\title{
REGULARIZATION OF TWO-TERM DIFFERENTIAL EQUATIONS WITH SINGULAR COEFFICIENTS BY QUASIDERIVATIVES
}

\author{
A. S. Goryunov and V. A. Mikhailets
}

UDC 517.984.5

We propose a regularization of the formal differential expression

$$
l(y)=i^{m} y^{(m)}(t)+q(t) y(t), \quad t \in(a, b),
$$

of order $m \geq 3$ by quasiderivatives. It is assumed that the distribution coefficient $q$ has the antiderivative $Q \in L([a, b] ; \mathbb{C})$. In the symmetric case $(Q=\bar{Q})$, we describe self-adjoint and maximal dissipative/accumulative extensions of the minimal operator and its generalized resolvents. In the general (nonself-adjoint) case, we establish the conditions of convergence for the resolvents of the analyzed operators in norm. The case where $m=2$ and $Q \in L_{2}([a, b] ; \mathbb{C})$ was studied earlier.

\section{Introduction}

In a finite interval $\mathcal{J}:=(a, b)$, we consider a formal differential expression of order $m$

$$
l(y)=i^{m} y^{(m)}(t)+q(t) y(t), \quad t \in \mathcal{J} .
$$

If $m=2$ and the coefficient $q \in L(\mathcal{J} ; \mathbb{R})$, then the differential equation $l(y)=f$ is the classical SturmLiouville problem, which is now studied fairly comprehensively. The state of the art of the theory of this equation is described in numerous monographs (see [1] and the references therein). After the appearance of the work [2], it turned out that various statements of this theory can be generalized to a much more general case

$$
q=Q^{\prime}, \quad Q \in L_{2}(\mathcal{J} ; \mathbb{C}),
$$

where the derivative is understood in the sense of distributions. Thus, in particular, this is true for a physically meaningful case where $q$ is the Radon measure on $\overline{\mathcal{J}}$ or has nonintegrable point singularities. Similar operators appeared much earlier in various problems of mathematical physics and were studied by many authors, mainly by the methods of the theory of operators (see [3] and the references therein). In particular, the case of differential expressions of any even order is studied in [4].

In this connection, it is of interest to study the problem of regularization of the differential expression (1) with singular coefficient $q \notin L(\mathcal{J} ; \mathbb{C})$ for any $m>2$. The present paper is devoted to the solution of this problem by using specially chosen quasiderivatives. Moreover, it is possible to weaken condition (2) to the following condition:

$$
q=Q^{\prime}, \quad Q \in L(\mathcal{J} ; \mathbb{C})=: L_{1} .
$$

Institute of Mathematics, Ukrainian National Academy of Sciences, Kyiv, Ukraine.

Translated from Ukrains'kyi Matematychnyi Zhurnal, Vol. 63, No. 9, pp. 1190-1205, September, 2011. Original article submitted June 21, 2011. 
The case of the general Sturm-Liouville expression

$$
l(y)=-\left(p(t) y^{\prime}\right)^{\prime}+q(t) y, \quad t \in \mathcal{J}
$$

with singular coefficients

$$
q=Q^{\prime}, \quad 1 / p, Q / p, Q^{2} / p \in L_{1}
$$

was studied earlier by the authors (from the similar positions) in [5] (see also [6]).

The paper is organized as follows:

In Sec. 2, we introduce a regularization of the formal differential expression (1) under assumption (3) and determine the corresponding maximal and minimal operators in the Hilbert space $L_{2}(\mathcal{J} ; \mathbb{C})=: L_{2}$.

In Sec.3, we establish sufficient conditions for the uniform resolvent approximation of extensions of the constructed minimal operator $L_{\min }$ by a family of operators from the same class, in particular, with smooth coefficients.

In Sec. 4, under the assumption that the minimal operator is symmetric, we describe all its self-adjoint, maximal dissipative, and maximal accumulative extensions in terms of homogeneous boundary conditions of the canonical form. These extensions are parametrized, respectively, by the unitary operators and by the contraction operators in $\mathbb{C}^{m}$. This parametrization is bijective and continuous.

In Sec. 5, we describe all generalized resolvents of the minimal operator outside the real axis.

The case of general symmetric quasidifferential operators was studied by the authors in $[7,8]$. For the preliminary version of the present paper, see [9].

\section{Regularization of the Singular Expression}

Consider a formal differential expression (1) of order $m \geq 3$ under conditions (3).

We now successively introduce the quasiderivatives:

$$
\begin{gathered}
D^{[k]} y(t):=y^{(k)}(t), \quad k=\overline{0, m-2}, \\
D^{[m-1]} y(t):=y^{(m-1)}(t)+i^{-m} Q(t) y(t), \\
D^{[m]} y(t):=\left(D^{[m-1]} y(t)\right)^{\prime}-i^{-m} Q(t) D^{[1]} y(t) .
\end{gathered}
$$

Under conditions (3), they are quasiderivatives in a Shin-Zettl sense (see [12], Sec. 1).

Thus, the formal expression (1) can be correctly defined as the quasidifferential Shin-Zettl expression

$$
l[y]:=i^{m} D^{[m]} .
$$

Definition 1. A solution of the Cauchy problem for the resolvent equation

$$
l[y]-\lambda y=f \in L_{2}, \quad\left(D^{[k]} y\right)(c)=\alpha_{k}, \quad k=\overline{0, m-1},
$$


where $c \in \overline{\mathcal{J}}$ and $\alpha_{k} \in \mathbb{C}, k=\overline{0, m-1}$, is defined as the first component of the solution of the Cauchy problem for the corresponding system of the first-order differential equations:

$$
w^{\prime}(t)=A_{\lambda}(t) w(t)+\varphi(t), \quad w(c)=\left(\alpha_{0}, \alpha_{1}, \ldots, \alpha_{m-1}\right),
$$

where the vector function $w(t):=\left(D^{[0]} y(t), D^{[1]} y(t), \ldots, D^{[m-1]} y(t)\right)$, the square matrix function

$$
A_{\lambda}(t):=\left(\begin{array}{cccccc}
0 & 1 & 0 & \ldots & 0 & 0 \\
0 & 0 & 1 & \ldots & 0 & 0 \\
\vdots & \vdots & \vdots & \ddots & \vdots & \vdots \\
0 & 0 & 0 & \ldots & 1 & 0 \\
-i^{-m} Q(t) & 0 & 0 & \ldots & 0 & 1 \\
i^{-m} \lambda & i^{-m} Q(t) & 0 & \ldots & 0 & 0
\end{array}\right) \in L_{1}^{m \times m}
$$

and the vector function $\varphi(t):=\left(0,0, \ldots, 0, i^{-m} f(t)\right)$ belongs to $L_{1}^{m}$.

Lemma 1. Under condition (3), the Cauchy problem (4) possesses a solution on $\overline{\mathcal{J}}$. Moreover, this solution is unique.

Proof. Problem (5), for $A_{\lambda}(\cdot) \in L_{1}^{m \times m}$, has a (unique) solution for any $c \in \overline{\mathcal{J}}$ and $\left(\alpha_{0}, \alpha_{2}, \ldots, \alpha_{m-1}\right) \in$ $\mathbb{C}^{m}$ by virtue of Theorem 1.2.1 in [1]. Hence, the statement of the lemma follows from Definition 1 and the indicated theorem.

The introduced quasidifferential expression $l[y]$ generates a maximal quasidifferential operator in the Hilbert space $L_{2}($ see $[11,12])$

$$
\begin{gathered}
L_{\text {max }}: y \rightarrow l[y], \\
\operatorname{Dom}\left(L_{\max }\right)=\left\{y \mid D^{[k]} y \in A C(\overline{\mathcal{J}}, \mathbb{C}), k=\overline{0, m-1}, D^{[m]} y \in L_{2}\right\} .
\end{gathered}
$$

The minimal quasidifferential operator is defined as the restriction of the operator $L_{\max }$ to the linear manifold

$$
\operatorname{Dom}\left(L_{\text {min }}\right):=\left\{y \in \operatorname{Dom}\left(L_{\text {max }}\right) \mid D^{[k]} y(a)=D^{[k]} y(b)=0, k=\overline{0, m-1}\right\} .
$$

The following assertion shows that the introduced operators are independent of the choice of the antiderivative $Q$ :

Lemma 2. If the chosen antiderivative $Q$ in equalities (1) and (3) is replaced by an arbitrary antiderivative

$$
\widetilde{Q}:=Q+c, \quad c \in \mathbb{C},
$$

then the operators $L_{\max }$ and $L_{\min }$ do not change. 
Proof. We show that the operator $L_{\max }=L_{\max }(Q)$ coincides with operator $\widetilde{L}_{\max }=L_{\max }(\tilde{Q})$. By $\widetilde{D}^{[0]} y, \widetilde{D}^{[1]} y, \ldots, \widetilde{D}^{[m]} y$ we denote the quasiderivatives corresponding to the antiderivative $\widetilde{Q}$ different from $Q$.

Let $y \in \operatorname{Dom}\left(L_{\max }\right)$. By direct calculations, we find

$$
\begin{gathered}
\widetilde{D}^{[0]} y=D^{[0]} y \in A C(\overline{\mathcal{J}}, \mathbb{C}), \\
\cdots \ldots \ldots \cdots \cdots \cdots \cdots \cdots \cdots \\
\widetilde{D}^{[m-2]} y=D^{[m-2]} y \in A C(\overline{\mathcal{J}}, \mathbb{C}), \\
\widetilde{D}^{[m-1]} y=D^{[m-1]} y+i^{-m} c \widetilde{D}^{[0]} y \in A C(\overline{\mathcal{J}}, \mathbb{C}), \\
\widetilde{D}^{[m]} y=D^{[m]} y \in L_{2} .
\end{gathered}
$$

This means that

$$
\operatorname{Dom}\left(L_{\max }\right) \subset \operatorname{Dom}\left(\widetilde{L}_{\text {max }}\right)=\left\{y \mid \widetilde{D}^{[k]} y \in A C(\overline{\mathcal{J}}, \mathbb{C}), k=\overline{0, m-1}, \widetilde{D}^{[m]} y \in L_{2}\right\}
$$

Similarly, we show that $\operatorname{Dom}\left(L_{\max }\right) \supset \operatorname{Dom}\left(\widetilde{L}_{\text {max }}\right)$ and, finally,

$$
\widetilde{L}_{\max } y=i^{m} \widetilde{D}^{[m]} y=i^{m} D^{[m]} y=L_{\max } y, \quad y \in \operatorname{Dom}\left(L_{\max }\right) .
$$

We now show that $\tilde{L}_{\text {min }}=L_{\text {min }}$.

Let $y \in \operatorname{Dom}\left(L_{\min }\right)$. Then

$$
\begin{gathered}
\widetilde{D}^{[0]} y(a)=D^{[0]} y(a)=0, \\
\widetilde{D}^{[0]} y(b)=D^{[0]} y(b)=0, \\
\ldots \ldots \ldots \ldots \ldots \ldots \\
\widetilde{D}^{[m-2]} y(a)=D^{[m-2]} y(a)=0, \\
\widetilde{D}^{[m-2]} y(b)=D^{[m-2]} y(b)=0, \\
\widetilde{D}^{[m-1]} y(a)=D^{[m-1]} y(a)+i^{-m} c \widetilde{D}^{[0]} y(a)=0+0=0, \\
\widetilde{D}^{[m-1]} y(b)=D^{[m-1]} y(b)+i^{-m} c \widetilde{D}^{[0]} y(b)=0+0=0 .
\end{gathered}
$$

This implies that $\operatorname{Dom}\left(L_{\text {min }}\right) \subset \operatorname{Dom}\left(\widetilde{L}_{\text {min }}\right)$. Similarly, we show that $\operatorname{Dom}\left(L_{\text {min }}\right) \supset \operatorname{Dom}\left(\tilde{L}_{\text {min }}\right)$. 
Since

$$
\tilde{L}_{\min } y=\tilde{L}_{\max } y=L_{\max } y=L_{\min } y
$$

on the functions $y \in \operatorname{Dom}\left(L_{\min }\right)$, the lemma is proved.

Parallel with (1), we consider the formally conjugate differential expression

$$
l^{+}(y)=i^{m} y^{(m)}(t)+\bar{q}(t) y(t)
$$

where the overbar denotes the operation of complex conjugation. By $L_{\max }^{+}$and $L_{\min }^{+}$we denote the maximal and minimal operators generated by this expression in the space $L_{2}$. Thus, by using the results for the general quasidifferential Shin-Zettl expressions from the monograph [12] and the arguments presented above, we arrive at the following theorem:

Theorem 1. The operators $L_{\min }, L_{\min }^{+}, L_{\max }$, and $L_{\max }^{+}$are densely defined and closed in the space $L_{2}$,

$$
L_{\min }^{*}=L_{\max }^{+}, \quad \text { and } \quad L_{\max }^{*}=L_{\min }^{+}
$$

If the function $q$ is real-valued, then $L_{\min }=L_{\min }^{+}$is a symmetric operator with deficiency index $(m, m)$ and, in addition,

$$
L_{\min }^{*}=L_{\max } \quad \text { and } \quad L_{\max }^{*}=L_{\min }
$$

\section{Approximation of the Resolvent}

Consider a family of quasidifferential expressions $l_{\varepsilon}[y]$ of the form (1) with coefficients $q_{\varepsilon}=Q_{\varepsilon}^{\prime}, Q_{\varepsilon} \in L_{1}$, $\varepsilon \in\left[0, \varepsilon_{0}\right]$. By $D_{\varepsilon}^{[0]} y, D_{\varepsilon}^{[1]} y, \ldots, D_{\varepsilon}^{[m]} y$ we denote the quasiderivatives of these expressions.

In the Hilbert space $L_{2}$ with norm $\|\cdot\|_{2}$, these expressions generate the operators $L_{\min }^{\varepsilon}$ and $L_{\max }^{\varepsilon}$ for any $\varepsilon$. Assume that the matrices $\alpha(\varepsilon), \beta(\varepsilon) \in \mathbb{C}^{m \times m}$ and the vectors

$$
\begin{aligned}
& \mathcal{Y}_{\varepsilon}(a):=\left\{D_{\varepsilon}^{[0]} y(a), D_{\varepsilon}^{[1]} y(a), \ldots, D_{\varepsilon}^{[m-1]} y(a)\right\} \in \mathbb{C}^{m}, \\
& \mathcal{Y}_{\varepsilon}(b):=\left\{D_{\varepsilon}^{[0]} y(b), D_{\varepsilon}^{[1]} y(b), \ldots, D_{\varepsilon}^{[m-1]} y(b)\right\} \in \mathbb{C}^{m} .
\end{aligned}
$$

For any fixed value of $\varepsilon$, we define the following operators:

$$
\begin{gathered}
L_{\varepsilon} y=l_{\varepsilon}[y] \\
\operatorname{Dom}\left(L_{\varepsilon}\right)=\left\{y \in \operatorname{Dom}\left(L_{\text {max }}^{\varepsilon}\right) \mid \alpha(\varepsilon) \mathcal{Y}_{\varepsilon}(a)+\beta(\varepsilon) \mathcal{Y}_{\varepsilon}(b)=0\right\} .
\end{gathered}
$$

It is clear that

$$
L_{\text {min }}^{\varepsilon} \subset L_{\varepsilon} \subset L_{\text {max }}^{\varepsilon}, \quad \varepsilon \in\left[0, \varepsilon_{0}\right]
$$


By $\rho(L)$ we denote the resolvent set of the operator $L$. Recall that the operators $L_{\varepsilon}$ converge as $\varepsilon \rightarrow 0+$ to the operator $L_{0}$ in a sense of uniform resolvent convergence, $L_{\varepsilon} \stackrel{R}{\rightarrow} L_{0}$, if there exists $\mu \in \mathbb{C}$ such that $\mu \in \rho\left(L_{0}\right), \quad \mu \in \rho\left(L_{\varepsilon}\right)$ for sufficiently small $\varepsilon$ and

$$
\left\|\left(L_{\varepsilon}-\mu\right)^{-1}-\left(L_{0}-\mu\right)^{-1}\right\| \rightarrow 0, \quad \varepsilon \rightarrow 0+.
$$

This definition is independent of the choice of $\mu \in \rho\left(L_{0}\right)$ [13].

We introduce the notation

$$
c^{\vee}(t):=\int_{a}^{t} c(s) d s .
$$

The main result of the present section is the following theorem:

Theorem 2. Assume that $\rho\left(L_{0}\right)$ is nonempty and the following conditions are satisfied as $\varepsilon \rightarrow 0+$ :

(i) $\left\|\left(Q_{\varepsilon}-Q_{0}\right)^{\vee}\right\|_{C} \rightarrow 0$;

(ii) $\alpha(\varepsilon) \longrightarrow \alpha(0)$ and $\beta(\varepsilon) \longrightarrow \beta(0)$.

Then $L_{\varepsilon} \stackrel{R}{\rightarrow} L_{0}$.

Remark 1. Clearly, the condition $\left\|Q_{\varepsilon}-Q_{0}\right\|_{1} \rightarrow 0, \varepsilon \rightarrow 0+$, is sufficient for the validity of condition (i). Since the set of infinitely differentiable finite functions $C_{0}^{\infty}(\mathcal{J}, \mathbb{C})$ is dense in the space $L_{1}$, it follows from Theorem 2 that every introduced operator $L_{0}$ with singular coefficient can be approximated in a sense of uniform resolvent convergence by a sequence of differential operators with coefficients from $C_{0}^{\infty}(\mathcal{J}, \mathbb{C})$.

The proof of Theorem 2 is based on one auxiliary result.

Following $[14,15]$, by $\mathcal{M}^{n}(\mathcal{J})=: \mathcal{M}^{n}, n \in \mathbb{N}$, we denote the class of all matrix functions

$$
R(\cdot ; \varepsilon):\left[0, \varepsilon_{0}\right] \rightarrow L_{1}^{n \times n}
$$

parametrized by the number $\varepsilon$ for which the solution of the Cauchy problem

$$
Z^{\prime}(t ; \varepsilon)=R(t ; \varepsilon) Z(t ; \varepsilon), \quad Z(a ; \varepsilon)=I_{n},
$$

satisfies the limiting relation

$$
\lim _{\varepsilon \rightarrow 0+}\left\|Z(\cdot ; \varepsilon)-I_{n}\right\|_{C}=0
$$

where $\|\cdot\|_{C}$ is the sup-norm.

The following general theorem is established in $[15,16]$ :

Theorem 3. Assume that, for the boundary-value problem

$$
\begin{gathered}
y^{\prime}(t ; \varepsilon)=A(t ; \varepsilon) y(t ; \varepsilon)+f(t ; \varepsilon), \quad t \in \mathcal{J}, \quad \varepsilon \in\left[0, \varepsilon_{0}\right], \\
U_{\varepsilon} y(\cdot ; \varepsilon)=0,
\end{gathered}
$$


where the matrix functions $A(\cdot, \varepsilon) \in L_{1}^{n \times n}$, the vector functions $f(\cdot, \varepsilon) \in L_{1}^{n}$, and the linear continuous operators

$$
U_{\varepsilon}: C\left(\overline{\mathcal{J}} ; \mathbb{C}^{n}\right) \rightarrow \mathbb{C}^{n}, \quad n \in \mathbb{N},
$$

the following conditions are satisfied:

(i) the homogeneous limiting boundary-value problem (7), (8) with $\varepsilon=0$ and $f(\cdot ; 0) \equiv 0$ possesses solely the trivial solution;

(ii) $A(\cdot ; \varepsilon)-A(\cdot ; 0) \in \mathcal{M}^{n}$;

(iii) $\left\|U_{\varepsilon}-U_{0}\right\| \rightarrow 0, \varepsilon \rightarrow 0+$.

Then, for sufficiently small $\varepsilon$, there exist Green matrices $G(t, s ; \varepsilon)$ of problems (7) and (8) and, in the square $\mathcal{J} \times \mathcal{J}$

$$
\|G(\cdot, \cdot ; \varepsilon)-G(\cdot, \cdot ; 0)\|_{\infty} \rightarrow 0, \quad \varepsilon \rightarrow 0+
$$

where $\|\cdot\|_{\infty}$ is the norm in the space $L_{\infty}$.

Remark 2. Condition (iii) of Theorem 3 cannot be replaced by a weaker condition of strong convergence of the operators $U_{\varepsilon} \stackrel{s}{\rightarrow} U_{0}$ [15]. However, it is easy to see that, for two-point boundary operators

$$
U_{\varepsilon} y:=B_{1}(\varepsilon) y(a)+B_{2}(\varepsilon) y(b), \quad B_{k}(\varepsilon) \in \mathbb{C}^{n \times n}, \quad k \in\{1,2\},
$$

both the condition of strong convergence and the condition of uniform convergence are equivalent to the condition

$$
\left\|B_{k}(\varepsilon)-B_{k}(0)\right\| \rightarrow 0, \quad \varepsilon \rightarrow 0+, \quad k \in\{1,2\}
$$

The presented definition of the class $\mathcal{M}^{n}$ is not constructive. There are different sufficient conditions for the matrix function $R(\cdot ; \varepsilon)$ to belong to the class $\mathcal{M}^{n}$. Thus, in particular, the results of the Levin's work [17] yield the following lemma:

Lemma 3. Let $R(\cdot ; \varepsilon):\left[0, \varepsilon_{0}\right] \rightarrow L_{1}^{n \times n}$. Suppose that one of the following four (nonequivalent) conditions is satisfied as $\varepsilon \rightarrow 0+$ :

$(\alpha) \quad\|R(\cdot ; \varepsilon)\|_{1}=O(1) ;$

( $\beta) \quad\left\|R^{\vee}(\cdot ; \varepsilon) R(\cdot ; \varepsilon)\right\|_{1} \rightarrow 0$

$(\gamma) \quad\left\|R(\cdot ; \varepsilon) R^{\vee}(\cdot ; \varepsilon)\right\|_{1} \rightarrow 0$

( $\delta) \quad\left\|R^{\vee}(\cdot ; \varepsilon) R(\cdot ; \varepsilon)-R(\cdot ; \varepsilon) R^{\vee}(\cdot ; \varepsilon)\right\|_{1} \rightarrow 0$.

Then the condition $\left\|R^{\vee}(\cdot ; \varepsilon)\right\|_{C} \rightarrow 0, \varepsilon \rightarrow 0+$, is equivalent to the inclusion $R(\cdot ; \varepsilon) \in \mathcal{M}^{n}$.

By using the following statement, we can reduce Theorem 2 to Theorem 3: 
Lemma 4. The function $y(t)$ is a solution of the boundary-value problem

$$
\begin{gathered}
l_{\varepsilon}[y](t)=f(t ; \varepsilon) \in L_{2}, \quad \varepsilon \in\left[0, \varepsilon_{0}\right], \\
\alpha(\varepsilon) \mathcal{Y}_{\varepsilon}(a)+\beta(\varepsilon) \mathcal{Y}_{\varepsilon}(b)=0
\end{gathered}
$$

if and only if the vector function

$$
w(t)=\left(D_{\varepsilon}^{[0]} y(t), D_{\varepsilon}^{[1]} y(t), \ldots, D_{\varepsilon}^{[m-1]} y(t)\right)
$$

is a solution of the boundary-value problem

$$
\begin{gathered}
w^{\prime}(t)=A(t ; \varepsilon) w(t)+\varphi(t ; \varepsilon), \\
\alpha(\varepsilon) w(a)+\beta(\varepsilon) w(b)=0,
\end{gathered}
$$

where the square matrix function

$$
A(\cdot ; \varepsilon):=\left(\begin{array}{cccccc}
0 & 1 & 0 & \ldots & 0 & 0 \\
0 & 0 & 1 & \ldots & 0 & 0 \\
\vdots & \vdots & \vdots & \ddots & \vdots & \vdots \\
0 & 0 & 0 & \ldots & 1 & 0 \\
-i^{-m} Q(\cdot ; \varepsilon) & 0 & 0 & \ldots & 0 & 1 \\
0 & i^{-m} Q(\cdot ; \varepsilon) & 0 & \ldots & 0 & 0
\end{array}\right) \in L_{1}^{m \times m}
$$

and $\varphi(\cdot ; \varepsilon):=\left(0,0, \ldots, 0, i^{-m} f(\cdot ; \varepsilon)\right)$ belongs to $L_{1}^{m}$.

Proof. Consider a system of equations

$$
\begin{gathered}
\left(D_{\varepsilon}^{[0]} y(t)\right)^{\prime}=D_{\varepsilon}^{[1]} y(t), \\
\left(D_{\varepsilon}^{[1]} y(t)\right)^{\prime}=D_{\varepsilon}^{[2]} y(t), \\
\ldots \ldots \ldots \ldots \ldots \ldots \ldots \\
\left(D_{\varepsilon}^{[m-3]} y(t)\right)^{\prime}=D_{\varepsilon}^{[m-2]} y(t), \\
\left(D_{\varepsilon}^{[m-2]} y(t)\right)^{\prime}=-i^{-m} Q_{\varepsilon}(t) D_{\varepsilon}^{[0]} y(t)+D_{\varepsilon}^{[m-1]} y(t), \\
\left(D_{\varepsilon}^{[m-1]} y(t)\right)^{\prime}=i^{-m} Q_{\varepsilon}(t) D_{\varepsilon}^{[1]} y(t)+i^{-m} f(t ; \varepsilon) .
\end{gathered}
$$


If $y(\cdot)$ is a solution of Eq. (10), then the definition of quasiderivatives implies that $y(\cdot)$ is a solution of this system. On the other hand, by setting

$$
w(t)=\left(D_{\varepsilon}^{[0]} y(t), D_{\varepsilon}^{[1]} y(t), \ldots, D_{\varepsilon}^{[m-1]} y(t)\right) \quad \text { and } \quad \varphi(t ; \varepsilon)=\left(0,0, \ldots, 0, i^{-m} f(t ; \varepsilon)\right),
$$

we can rewrite this system in the form of Eq. (12).

Since $\mathcal{Y}_{\varepsilon}(a)=w(a)$ and $\mathcal{Y}_{\varepsilon}(b)=w(b)$, we immediately conclude that the boundary conditions (11) are equivalent to the boundary conditions (13).

By virtue of Lemma 4, the assumption that

$(\epsilon)$ the homogeneous boundary-value problem

$$
D_{0}^{[m]} y(t)=0, \quad \alpha(0) \mathcal{Y}_{0}(a)+\beta(0) \mathcal{Y}_{0}(b)=0
$$

possesses solely the trivial solution

implies that the homogeneous boundary-value problem

$$
w^{\prime}(t)=A(t ; \varepsilon) w(t), \quad \alpha(\varepsilon) w(a)+\beta(\varepsilon) w(b)=0
$$

also possesses solely the trivial solution for sufficiently small $\varepsilon$.

Lemma 5. Assume that the Green matrix

$$
G(t, s, \varepsilon)=\left(g_{i j}(t, s)\right)_{i, j=1}^{m} \in L_{\infty}^{m \times m}
$$

exists for problem (12), (13) for sufficiently small $\varepsilon$. Then there exists the Green function $\Gamma(t, s ; \varepsilon)$ of the semihomogeneous boundary-value problem (10), (11) and

$$
\Gamma(t, s ; \varepsilon)=i^{-m} g_{1 m}(t, s ; \varepsilon) \quad \text { almost everywhere. }
$$

Proof. By the definition of the Green matrix, the unique solution of problem (12), (13) can be represented in the form

$$
w_{\varepsilon}(t)=\int_{a}^{b} G(t, s ; \varepsilon) \varphi(s ; \varepsilon) d s, \quad t \in \overline{\mathcal{J}}
$$

By virtue of Lemma 4, the last equality takes the form

$$
D_{\varepsilon}^{[0]} y_{\varepsilon}(t)=\int_{a}^{b} g_{1 m}(t, s ; \varepsilon) i^{-m} f(s ; \varepsilon) d s,
$$




$$
\begin{gathered}
D_{\varepsilon}^{[1]} y_{\varepsilon}(t)=\int_{a}^{b} g_{2 m}(t, s ; \varepsilon) i^{-m} f(s ; \varepsilon) d s, \\
\ldots \ldots \ldots \ldots \ldots \ldots \ldots \ldots \ldots \ldots \ldots \ldots \ldots \\
D_{\varepsilon}^{[m-1]} y_{\varepsilon}(t)=\int_{a}^{b} g_{m m}(t, s ; \varepsilon) i^{-m} f(s ; \varepsilon) d s,
\end{gathered}
$$

where $y_{\varepsilon}(\cdot)$ is the unique solution of problem (10), (11). This yields the assertion of Lemma 5.

Proof of Theorem 2. By virtue of condition (i) of Theorem 2, without loss of generality, we can assume that $0 \in \rho\left(L_{0}\right)$.

We now show that $\sup _{\|f\|_{2}=1}\left\|L_{\varepsilon}^{-1} f-L_{0}^{-1} f\right\| \rightarrow 0, \varepsilon \rightarrow 0+$.

The equation $L_{\varepsilon}^{-1} f=y_{\varepsilon}$ is equivalent to the equation $L_{\varepsilon} y_{\varepsilon}=f$, i.e., $y_{\varepsilon}$ is a solution of problem (10), (11). It follows from the inclusion $0 \in \rho\left(L_{0}\right)$ that the assumption $(\epsilon)$ is true.

Denote $r(\cdot ; \varepsilon):=i^{-m} Q(\cdot ; \varepsilon)-i^{-m} Q(\cdot ; 0)$. Then

$$
\begin{gathered}
A(\cdot ; \varepsilon)-A(\cdot ; 0)=\left(\begin{array}{cccccc}
0 & 0 & 0 & \ldots & 0 & 0 \\
0 & 0 & 0 & \ldots & 0 & 0 \\
\vdots & \vdots & \vdots & \ddots & \vdots & \vdots \\
-r(\cdot ; \varepsilon) & 0 & 0 & \ldots & 0 & 0 \\
0 & r(\cdot ; \varepsilon) & 0 & \ldots & 0 & 0
\end{array}\right), \\
(A(\cdot ; \varepsilon)-A(\cdot ; 0))^{\vee}=\left(\begin{array}{ccccccc}
0 & 0 & 0 & \ldots & 0 & 0 \\
0 & 0 & 0 & \ldots & 0 & 0 \\
\vdots & \vdots & \vdots & \ddots & \vdots & \vdots \\
-r^{\vee}(\cdot ; \varepsilon) & 0 & 0 & \ldots & 0 & 0 \\
0 & r^{\vee}(\cdot ; \varepsilon) & 0 & \ldots & 0 & 0
\end{array}\right),
\end{gathered}
$$

where the matrix function $A(\cdot ; \varepsilon)$ is defined by relation (14).

It is easy to see that

$$
(A(\cdot ; \varepsilon)-A(\cdot ; 0))(A(\cdot ; \varepsilon)-A(\cdot ; 0))^{\vee}=(A(\cdot ; \varepsilon)-A(\cdot ; 0))^{\vee}(A(\cdot ; \varepsilon)-A(\cdot ; 0)) .
$$

Hence, the matrix function $A(\cdot ; \varepsilon)-A(\cdot ; 0)$, for $m \geq 3$, satisfies condition $(\delta)$ of Lemma 3 .

It is clear that the condition $\left\|(A(\cdot ; \varepsilon)-A(\cdot ; 0))^{\vee}\right\|_{C} \rightarrow 0, \varepsilon \rightarrow 0+$, is equivalent to condition (i) of Theorem 2. Therefore, it follows from Lemma 3 that the conditions of Theorem 3 are satisfied for problem (12), (13). 
This means that there exist Green matrices $G(t, s ; \varepsilon)$ of problem (12), (13) and the limit relation (9) is true. By virtue of Lemma 5, this yields the limit equality

$$
\|\Gamma(\cdot, \cdot ; \varepsilon)-\Gamma(\cdot, \cdot ; 0)\|_{\infty} \rightarrow 0, \quad \varepsilon \rightarrow 0+
$$

Then

$$
\begin{aligned}
\left\|L_{\varepsilon}^{-1}-L_{0}^{-1}\right\| & =\sup _{\|f\|_{2}=1}\left\|\int_{a}^{b}[\Gamma(t, s ; \varepsilon)-\Gamma(t, s ; 0)] f(s) d s\right\|_{2} \\
& \leq(b-a)^{1 / 2} \sup _{\|f\|_{2}=1}\left\|\int _ { a } ^ { b } \left|\Gamma(t, s ; \varepsilon)-\Gamma(t, s ; 0)\|f(s) \mid d s\|_{C}\right.\right. \\
& \leq(b-a)\|\Gamma(\cdot, \cdot ; \varepsilon)-\Gamma(\cdot, \cdot ; 0)\|_{\infty} \rightarrow 0, \quad \varepsilon \rightarrow 0+,
\end{aligned}
$$

which proves Theorem 2.

\section{Extensions of the Symmetric Minimal Operator}

In what follows, we assume that the functions $q$ and, hence, $Q$ are real-valued. This condition implies the formal self-adjointness of the expression $l[y]$ (see [12]) and, according to Theorem 1 , the property of symmetry of the operator $L_{\min }$. Thus, it is of interest to study the problem of description (with the help of homogeneous boundary conditions) of some classes of extensions (self-adjoint, maximal dissipative, and maximal accumulative) of the symmetric operator $L_{\min }$ in the Hilbert space $L_{2}$. To answer to this question, we use the notion of space of boundary values.

Definition 2. Let $L$ be a closed symmetric operator in the Hilbert space $\mathcal{H}$ with equal (finite or infinite) deficiency indices. A triple $\left(H, \Gamma_{1}, \Gamma_{2}\right)$, where $H$ is an auxiliary Hilbert space and $\Gamma_{1}$ and $\Gamma_{2}$ are linear mappings of $\operatorname{Dom}\left(L^{*}\right)$ into $H$, is called a space of boundary values of the symmetric operator $L$ if

(i) for any $f, g \in \operatorname{Dom}\left(L^{*}\right)$,

$$
\left(L^{*} f, g\right)_{\mathcal{H}}-\left(f, L^{*} g\right)_{\mathcal{H}}=\left(\Gamma_{1} f, \Gamma_{2} g\right)_{H}-\left(\Gamma_{2} f, \Gamma_{1} g\right)_{H}
$$

(ii) for any vectors $f_{1}, f_{2} \in H$, there exists a vector $f \in \operatorname{Dom}\left(L^{*}\right)$ such that $\Gamma_{1} f=f_{1}$ and $\Gamma_{2} f=f_{2}$.

It follows from the definition of the space of boundary values that $f \in \operatorname{Dom}(L)$ if and only if $\Gamma_{1} f=$ $\Gamma_{2} f=0$. A space of boundary values exists for any symmetric operator with equal nonzero deficiency indices (see [18-20]). It is always not unique.

The following lemma is the key result for the remaining part of the present paper: 
Basic Lemma. Let $\Gamma_{1}$ and $\Gamma_{2}$ be linear mappings from $\operatorname{Dom}\left(L_{\max }\right)$ into $\mathbb{C}^{m}$ such that

$$
\Gamma_{1} y:=i^{2 n}\left(\begin{array}{c}
-D^{[2 n-1]} y(a) \\
\ldots \ldots \ldots \ldots \ldots \\
(-1)^{n} D^{[n]} y(a) \\
D^{[2 n-1]} y(b) \\
\ldots \ldots \ldots \ldots . \\
(-1)^{n-1} D^{[n]} y(b)
\end{array}\right), \quad \Gamma_{2} y:=\left(\begin{array}{c}
D^{[0]} y(a) \\
\ldots \ldots \ldots \\
D^{[n-1]} y(a) \\
D^{[0]} y(b) \\
\ldots \ldots \ldots \\
D^{[n-1]} y(b)
\end{array}\right)
$$

for $m=2 n, n \geq 2$, and

$$
\Gamma_{1} y:=i^{2 n+1}\left(\begin{array}{c}
-D^{[2 n]} y(a) \\
\ldots \ldots \ldots \ldots \ldots \ldots \\
(-1)^{n} D^{[n+1]} y(a) \\
D^{[2 n]} y(b) \\
\ldots \ldots \ldots \ldots \ldots \ldots \\
(-1)^{n-1} D^{[n+1]} y(b) \\
\alpha D^{[n]} y(b)+\beta D^{[n]} y(a)
\end{array}\right), \quad \Gamma_{2} y:=\left(\begin{array}{c}
D^{[0]} y(a) \\
\ldots \ldots \ldots \ldots \\
D^{[n-1]} y(a) \\
D^{[0]} y(b) \\
\ldots \ldots \ldots \ldots \\
D^{[n-1]} y(b) \\
\gamma D^{[n]} y(b)+\delta D^{[n]} y(a)
\end{array}\right)
$$

for $m=2 n+1, n \in \mathbb{N}$, where

$$
\alpha=1, \quad \beta=1, \quad \gamma=\frac{(-1)^{n}}{2}+i, \quad \text { and } \quad \delta=\frac{(-1)^{n+1}}{2}+i
$$

Then the triple $\left(\mathbb{C}^{m}, \Gamma_{1}, \Gamma_{2}\right)$ is the space of boundary values of the operator $L_{\min }$.

Remark 3. The presented values of the coefficients can be replaced by arbitrary families of numbers satisfying the system of equations

$$
\begin{gathered}
\alpha \bar{\gamma}+\bar{\alpha} \gamma=(-1)^{n}, \\
\beta \bar{\delta}+\bar{\beta} \delta=(-1)^{n+1}, \\
\alpha \bar{\delta}+\bar{\beta} \gamma=0, \\
\beta \bar{\gamma}+\bar{\alpha} \delta=0, \\
\alpha \delta-\beta \gamma \neq 0 .
\end{gathered}
$$


By $L_{K}$ we denote the restriction of the operator $L_{\max }$ to the set of functions $y(t) \in \operatorname{Dom}\left(L_{\max }\right)$ satisfying the homogeneous boundary condition of the canonical form

$$
(K-I) \Gamma_{1} y+i(K+I) \Gamma_{2} y=0,
$$

where $K$ is a bounded operator in the Hilbert space $\mathbb{C}^{m}$.

Similarly, let $L^{K}$ be the restriction of the operator $L_{\max }$ to the set of functions $y(t) \in \operatorname{Dom}\left(L_{\max }\right)$ satisfying the homogeneous boundary condition of the canonical form

$$
(K-I) \Gamma_{1} y-i(K+I) \Gamma_{2} y=0,
$$

where $K$ is a bounded operator in the Hilbert space $\mathbb{C}^{m}$.

The basic lemma, together with the results presented in [18], yields the following description of the self-adjoint extensions of $L_{\min }$ :

Theorem 4. Every $L_{K}$, where $K$ is a unitary operator in the Hilbert space $\mathbb{C}^{m}$, is a self-adjoint extension of the operator $L_{\min }$. Conversely, for any self-adjoint extension $\tilde{L}$ of the operator $L_{\min }$, one can find a unitary operator $K$ such that $\widetilde{L}=L_{K}$. The correspondence between the unitary operators $\{K\}$ and the extensions $\{\widetilde{L}\}$ is bijective.

Remark 4. It follows from Theorems 2 and 4 that the mapping $K \rightarrow L_{K}$ is not only bijective but also continuous. More precisely, if the unitary operators $K_{n}$ converge to the operator $K$ in norm, then

$$
\left\|\left(L_{K}-\lambda\right)^{-1}-\left(L_{K_{n}}-\lambda\right)^{-1}\right\| \rightarrow 0, \quad n \rightarrow \infty, \quad \operatorname{Im} \lambda \neq 0 .
$$

Moreover, since the set of unitary operators in the finite-dimensional space $\mathbb{C}^{m}$ is compact in the metric of the operator norm, the converse assertion is also true, i.e., the mapping

$$
K \rightarrow\left(L_{K}-\lambda\right)^{-1}, \quad \operatorname{Im} \lambda \neq 0,
$$

is a homeomorphism for any fixed $\lambda \in \mathbb{C} \backslash \mathbb{R}$.

We now recall the well-known definition:

Definition 3. A densely defined linear operator $L$ in the complex Hilbert space $\mathcal{H}$ is called dissipative (accumulative) if

$$
\operatorname{Im}(L f, f)_{\mathcal{H}} \geq 0 \quad(\leq 0), \quad f \in \operatorname{Dom}(L),
$$

and maximal dissipative (maximal accumulative) if, in addition, the operator $L$ does not have nontrivial dissipative (accumulative) extensions in the space $\mathcal{H}$.

In particular, any symmetric operator is dissipative and accumulative and any self-adjoint operator is both maximal dissipative and maximal accumulative. Hence, for the symmetric quasidifferential operator $L_{\min }$, we can pose the problem of description of all its maximal dissipative and maximal accumulative solutions. According to the Phillips theorem [18,21], every dissipative extension of a symmetric operator and every accumulative extension of this operator are restrictions of its adjoint operator. Thus, any maximal dissipative or maximal accumulative extension of the operator $L_{\min }$ is a restriction of the operator $L_{\max }$. 
The following theorem gives the parametric description of all maximal dissipative extensions of the symmetric quasidifferential operator $L_{\min }$ :

Theorem 5. Every $L_{K}$, where $K$ is a contraction in the space $\mathbb{C}^{m}$, is a maximal dissipative extension $L_{K}$ of the operator $L_{\min }$. Conversely, for any maximal dissipative extension $\widetilde{L}$ of the operator $L_{\min }$, one can find a contraction $K$ such that $\widetilde{L}=L_{K}$. The correspondence between the contractions $\{K\}$ and the extensions $\{\widetilde{L}\}$ is bijective.

The following theorem gives a parametric description of all maximal accumulative extensions of the symmetric quasidifferential operator $L_{\min }$ :

Theorem 6. Every $L^{K}$, where $K$ is a contraction in the space $\mathbb{C}^{m}$, is a maximal accumulative extension $L^{K}$ of the operator $L_{\min }$. Conversely, for any maximal accumulative extension $\tilde{L}$ of the operator $L_{\min }$, one can find a contraction $K$ such that $\widetilde{L}=L^{K}$. The correspondence between the contractions $\{K\}$ and the extensions $\{\tilde{L}\}$ is bijective.

Remark 5. The mappings

$$
\begin{gathered}
K \rightarrow\left(L_{K}-\lambda\right)^{-1}, \quad \operatorname{Im} \lambda<0, \\
K \rightarrow\left(L^{K}-\lambda\right)^{-1}, \quad \operatorname{Im} \lambda>0,
\end{gathered}
$$

are homeomorphisms for any fixed $\lambda$ (see Remark 4).

We now proceed to the proof of the formulated results. Prior to proving the basic lemma, we present two more lemmas, which are, in fact, special cases of the corresponding assertions for the general quasidifferential expressions (see [12]).

Lemma 6. Let $y, z \in \operatorname{Dom}\left(L_{\max }\right)$. Then

$$
\int_{a}^{b}\left(D^{[m]} y \cdot \bar{z}-y \cdot \overline{D^{[m]} z}\right) d x=\sum_{k=1}^{m}(-1)^{k-1} D^{[m-k]} y \cdot \overline{D^{[k-1]} z} \mid \begin{aligned}
& x=b \\
& x=a
\end{aligned} .
$$

Lemma 7. For arbitrary collections of complex numbers $\left\{\alpha_{0}, \alpha_{1}, \ldots, \alpha_{m-1}\right\}$ and $\left\{\beta_{0}, \beta_{1}, \ldots, \beta_{m-1}\right\}$, there exist a function $y \in \operatorname{Dom}\left(L_{\max }\right)$ such that

$$
D^{[k]} y(a)=\alpha_{k}, \quad D^{[k]} y(b)=\beta_{k}, \quad k=0,1, \ldots, m-1 .
$$

Proof of the Basic Lemma. It suffices to show that the triple $\left(\mathbb{C}^{m}, \Gamma_{1}, \Gamma_{2}\right)$ satisfies conditions (i) and (ii) in the definition of the space of boundary values with $\mathcal{H}=L_{2}$. According to Theorem $1, L_{\min }^{*}=L_{\max }$. By virtue of Lemma 6, we get

$$
\left(L_{\max } y, z\right)-\left(y, L_{\max } z\right)=\left.i^{m} \sum_{k=1}^{m}(-1)^{k-1} D^{[m-k]} y \cdot \overline{D^{[k-1]} z}\right|_{\substack{x=b \\ x=a}} .
$$


However, it is easy to see that, in the case $m=2 n$, we have

$$
\left(\Gamma_{1} y, \Gamma_{2} z\right)=i^{2 n} \sum_{k=1}^{n}(-1)^{k-1} D^{[2 n-k]} y \cdot \overline{D^{[k-1]} z} \mid \begin{aligned}
& x=b \\
& x=a
\end{aligned}
$$

and

$$
\left(\Gamma_{2} y, \Gamma_{1} z\right)=i^{2 n} \sum_{k=n+1}^{2 n}(-1)^{k} D^{[2 n-k]} y \cdot \overline{D^{[k-1]} z} \mid \begin{aligned}
& x=b \\
& x=a
\end{aligned} .
$$

This means that condition (i) is satisfied. Condition (ii) directly follows from Lemma 7.

For $m=2 n+1$, we introduce the notation

$$
\begin{aligned}
& \Gamma_{1}=:\left(\Gamma_{1 a}, \Gamma_{1 b}, \Gamma_{1 a b}\right), \\
& \Gamma_{2}=:\left(\Gamma_{2 a}, \Gamma_{2 b}, \Gamma_{2 a b}\right),
\end{aligned}
$$

where

$$
\begin{gathered}
\Gamma_{1 a}=i^{2 n+1}\left(-D^{[2 n]} y(a), \ldots,(-1)^{n} D^{[n+1]} y(a)\right), \\
\Gamma_{1 b}=i^{2 n+1}\left(D^{[2 n]} y(b), \ldots,(-1)^{n+1} D^{[n+1]} y(a)\right), \\
\Gamma_{1 a b}=i^{2 n+1}\left(\alpha D^{[n]} y(b)+\beta D^{[n]} y(a)\right), \\
\Gamma_{2 a}=\left(D^{[0]} y(a), \ldots, D^{[n-1]} y(a)\right) \\
\Gamma_{2 b}=\left(D^{[0]} y(b), \ldots, D^{[n-1]} y(b)\right) \\
\Gamma_{2 a b}=\gamma D^{[n]} y(b)+\delta D^{[n]} y(a) .
\end{gathered}
$$

It is easy to see that

$$
\begin{aligned}
& \left(\Gamma_{1 a} y, \Gamma_{2 a} z\right)=i^{2 n+1} \sum_{k=1}^{n}(-1)^{k-1} D^{[2 n-k]} y(a) \cdot \overline{D^{[k-1]} z(a)}, \\
& \left(\Gamma_{2 a} y, \Gamma_{1 a} z\right)=i^{2 n+1} \sum_{k=n+2}^{2 n+1}(-1)^{k} D^{[2 n-k]} y(a) \cdot \overline{D^{[k-1]} z(a)},
\end{aligned}
$$




$$
\begin{gathered}
\left(\Gamma_{1 b} y, \Gamma_{2 b} z\right)=i^{2 n+1} \sum_{k=1}^{n}(-1)^{k-1} D^{[2 n-k]} y(b) \cdot \overline{D^{[k-1]} z(b)}, \\
\left(\Gamma_{2 b} y, \Gamma_{1 b} z\right)=i^{2 n+1} \sum_{k=n+2}^{2 n+1}(-1)^{k} D^{[2 n-k]} y(b) \cdot \overline{D^{[k-1]} z(b)}, \\
\left(\Gamma_{1 a b} y, \Gamma_{2 a b} z\right)-\left(\Gamma_{2 a b} y, \Gamma_{1 a b} z\right)=i^{2 n+1}(-1)^{n}\left(D^{[n]} y(b) \cdot \overline{D^{[n]} z(b)}-D^{[n]} y(a) \cdot \overline{D^{[n]} z(a)}\right) .
\end{gathered}
$$

It follows from these relations that condition (i) of Definition 2 is satisfied. Condition (ii) follows from Lemma 7 and the last relation in (15).

Proof of Theorems 4-6. The assertions of the theorems follow from the basic lemma and Theorem 1.6 in [18] (Chap. 3) for the space of boundary values of an abstract symmetric operator.

\section{Generalized Resolvents}

We now recall the following well-known definition:

Definition 4. A generalized resolvent of a closed symmetric operator $L$ is defined as an operator function $R_{\lambda}$ of the complex parameter $\lambda \in \mathbb{C} \backslash \mathbb{R}$ which admits the following representation:

$$
R_{\lambda} f=P^{+}\left(L^{+}-\lambda I^{+}\right)^{-1} f, \quad f \in \mathcal{H}
$$

where $L^{+}$is an arbitrary self-adjoint extension of the operator $L$ onto the space $\mathcal{H}^{+}$, generally speaking, wider than $\mathcal{H}, I^{+}$is the identity operator in $\mathcal{H}^{+}$, and $P^{+}$is the operator of orthogonal projection of $\mathcal{H}^{+}$onto $\mathcal{H}$.

The operator function $R_{\lambda}(\operatorname{Im} \lambda \neq 0)$ is a generalized resolvent of the symmetric operator $L$ if and only if

$$
\left(R_{\lambda} f, g\right)_{\mathcal{H}}=\int_{-\infty}^{+\infty} \frac{d\left(F_{\mu} f, g\right)}{\mu-\lambda}, \quad f, g \in \mathcal{H}
$$

where $F_{\mu}$ is the generalized spectral function of the operator $L$. This means that the operator function $F_{\mu}$, $\mu \in \mathbb{R}$, has the following properties [22]:

$\left(1^{\circ}\right)$ for $\mu_{2}>\mu_{1}$, the difference $F_{\mu_{2}}-F_{\mu_{1}}$ is a bounded nonnegative operator;

$\left(2^{\circ}\right) \quad F_{\mu+}=F_{\mu}$ for all real $\mu$;

$\left(3^{\circ}\right)$ for any $x \in \mathcal{H}$,

$$
\lim _{\mu \rightarrow-\infty}\left\|F_{\mu} x\right\|_{\mathcal{H}}=0 \quad \text { and } \quad \lim _{\mu \rightarrow+\infty}\left\|F_{\mu} x-x\right\|_{\mathcal{H}}=0
$$

The following result belongs to Bruk [20]: 
Let $H$ be an auxiliary separable Hilbert space. By $\left\{X, X^{\prime}\right\}$ we denote an ordered couple from $X, X^{\prime} \in H$. The couples $\left\{X, X^{\prime}\right\}$ are regarded as elements of the space $H \oplus H$. Assume that there exists a linear operator $\gamma$ mapping the domain of definition $\operatorname{Dom}\left(L^{*}\right)$ of the operator $L^{*}$ adjoint to $L$ onto $H \oplus H$ and such that the following equality holds:

$$
\left(L^{*} x, y\right)-\left(x, L^{*} y\right)=\left(X^{\prime}, Y\right)_{H}-\left(X, Y^{\prime}\right)_{H},
$$

where $x, y \in \operatorname{Dom}(L),\left\{X, X^{\prime}\right\}=\gamma x$, and $\left\{Y, Y^{\prime}\right\}=\gamma y$.

Theorem 7. There exists a one-to-one correspondence between the generalized resolvents of the operator $L$ and the boundary-value problems

$$
\begin{gathered}
L^{*} y=\lambda y+h, \\
(K(\lambda)-I) Y^{\prime} \mp i(K(\lambda)+I) Y=0,
\end{gathered}
$$

where $\left\{Y, Y^{\prime}\right\}=\gamma y, h \in \mathcal{H}, \lambda$ is a complex number, the sign " + " in the boundary condition is taken for the values of $\lambda$ from the upper half plane, the sign " - " is taken for the values of $\lambda$ from the lower half plane, and $K(\lambda)$ is a given operator function from $H$ regular in the upper half plane and such that $\|K(\lambda)\| \leq 1$; for the values of $\lambda$ from the lower half plane, $K(\lambda):=K^{*}(\bar{\lambda})$.

Every solution of the problem specifies a generalized resolvent of the operator $L$ and, conversely, every generalized resolvent of the operator $L$ is defined by a solution of this problem.

This theorem enables one to describe all generalized resolvents of the symmetric operator $L_{\text {min }}$ outside the real axis.

The parametric inner description of all generalized resolvents of the operator is given by the following theorem:

Theorem 8. The following statements are true:

(i) every generalized resolvent $R_{\lambda}$ of the operator $L_{\min }$ in the half plane $\operatorname{Im} \lambda<0$ is given by the formula $R_{\lambda} h=y$, where $y$ is a solution of the boundary-value problem

$$
\begin{gathered}
l[y]=\lambda y+h, \\
(K(\lambda)-I) \Gamma_{[1]} f+i(K(\lambda)+I) \Gamma_{[2]} f=0,
\end{gathered}
$$

where $h(x) \in L_{2}(\mathcal{J}, \mathbb{C})$ and $K(\lambda)$ is an operator function regular in the lower half plane of the space $\mathbb{C}^{2}$ and such that $\|K(\lambda)\| \leq 1$;

(ii) in the half plane $\operatorname{Im} \lambda>0$, every generalized resolvent of the operator $L_{\min }$ is given by the formula $R_{\lambda} h=y$, where $y$ is a solution of the boundary-value problem

$$
\begin{gathered}
l[y]=\lambda y+h, \\
(K(\lambda)-I) \Gamma_{[1]} f-i(K(\lambda)+I) \Gamma_{[2]} f=0,
\end{gathered}
$$


where $h(x) \in L_{2}(\mathcal{J}, \mathbb{C})$ and $K(\lambda)$ is an operator function regular in the upper half plane of the space $\mathbb{C}^{2}$ and such that $\|K(\lambda)\| \leq 1$.

The indicated parametrization of the generalized resolvents by the operator functions $K$ is bijective.

Proof. By virtue of the basic lemma, the auxiliary separable Hilbert space $\mathbb{C}^{m}$ and the operator $\gamma y=$ $\left\{\Gamma_{[1]} y, \Gamma_{[2]} y\right\}$ mapping $\operatorname{Dom}\left(L_{\min }\right)$ onto $\mathbb{C}^{m} \oplus \mathbb{C}^{m}$ satisfy the conditions of Theorem 7.

Thus, the assertion of Theorem 8 follows from Theorem 7 .

\section{REFERENCES}

1. A. Zettl, Sturm-Liouville Theory, American Mathematical Society, Providence, RI (2005).

2. A. M. Savchuk and A. A. Shkalikov, "Sturm-Liouville operators with singular potentials," Mat. Zametki, 66, No. 6, 897-912 (1999).

3. S. Albeverio, F. Gesztesy, R. Høegh-Krohn, and H. Holden, Solvable Models in Quantum Mechanics, Springer, New York (1988).

4. V. A. Mikhailets and V. M. Molyboga, "Singularly perturbed periodic and semiperiodic differential operators," Ukr. Mat. Zh., 59, No. 6, 785-797 (2007); English translation: Ukr. Math. J., 59, No. 6, 858-873 (2007).

5. A. S. Goriunov and V. A. Mikhailets, "Regularization of singular Sturm-Liouville equations," Meth. Funct. Anal. Topol., No. 2, 120130 (2010).

6. A. S. Goryunov and V. A. Mikhailets, "Resolvent convergence of Sturm-Liouville operators with singular potentials," Mat. Zametki, 87, No. 2, 311-315 (2010).

7. A. S. Goryunov and V. A. Mikhailets, "On the extensions of symmetric quasidifferential operators of even order," Dopov. Nats. Akad. Nauk Ukr., No. 4, 19-24 (2009).

8. A. S. Goryunov and V. A. Mikhailets, "On the extensions of symmetric quasidifferential operators of odd order," Dopov. Nats. Akad. Nauk Ukr., No. 9, 27-31 (2009).

9. A. S. Goryunov and V. A. Mikhailets, "Regularization of binomial differential equations with singular coefficients," in: Proc. of the Institute of Mathematics, Ukrainian National Academy of Sciences [in Russian], Kyiv, 7, No. 1 (2010), pp.49-67; arXiv:1106.3275 [math.FA]

10. D. Shin, "On quasidifferential operators in Hilbert spaces," Mat. Sb., 13(55), No. 1, 39-70 (1943).

11. A. Zettl, "Formally self-adjoint quasidifferential operators," Rocky Mountain J. Math., 5, No. 3, $453-474$ (1975).

12. W. N. Everitt and L. Markus, Boundary-Value Problems and Symplectic Algebra for Ordinary Differential and Quasidifferential Operators, American Mathematical Society, Providence, RI (1999).

13. T. Kato, Perturbation Theory for Linear Operators, Springer, Berlin (1966).

14. V. A. Mikhailets and N. V. Reva, "Generalizations of the Kiguradze theorem on well-posedness of linear boundary-value problems," Dopov. Nats. Akad. Nauk Ukr., No. 9, 23-27 (2008).

15. V. A. Mikhailets and N. V. Reva, "Continuous dependence of the solutions of general boundary-value problems on the parameter," in: Proc. of the Institute of Mathematics, Ukrainian National Academy of Sciences [in Russian], Kyiv, 5, No. 1 (2008), pp. $227-239$.

16. T. I. Kodlyuk, V. A. Mikhailets, and N. V. Reva, Continuous Dependence of the Solutions of One-Dimensional Boundary-Value Problems on the Parameter, arXiv:1106.4174 [math.AP]

17. A. Yu. Levin, "Limit transition for the nonsingular systems $\dot{X}=A_{n}(t) X$," Dokl. Akad. Nauk SSSR, 176, No. 4, 774-777 (1967).

18. V. I. Gorbachuk and M. L. Gorbachuk, Boundary-Value Problems for Differential-Operator Equations [in Russian], Naukova Dumka, Kiev (1984).

19. A. N. Kochubei, "On the extensions of symmetric operators and symmetric binary relations," Mat. Zametki, 17, No. 1, 41-48 (1975).

20. V. M. Bruk, "On one class of boundary-value problems with spectral parameter in the boundary condition," Mat. Sb., 100(142), No. 2(6), 210-216 (1976).

21. R. S. Phillips, "Dissipative operators and hyperbolic systems of partial differential equations," Trans. Amer. Math. Soc., 90, 193-254 (1959).

22. N. I. Akhiezer and I. M. Glazman, Theory of Linear Operators in Hilbert Spaces [in Russian], Nauka, Moscow (1966). 